Vol. 14, e14005, p. 1-12, 2021

ISSN 1984-3534

\title{
A RELAÇÃO ENTRE O TEMPO DE ABERTURA DO COMÉRCIO, FATURAMENTO DAS LOJAS E EXPOSIÇÃO DOS CLIENTES AO COVID-19 COM USO DE TEORIA DE FILAS ${ }^{1}$
}

\author{
Rodrigo Luiz Gigante ${ }^{a *}$, Henrique Ewbank de Miranda Vieira ${ }^{a}$, Anibal Tavarez de \\ Azevedo ${ }^{\mathrm{b}}$ \\ ${ }^{\mathrm{a}}$ Centro Universitário Facens Sorocaba-SP, Brasil \\ ${ }^{b}$ Faculdade de Ciências Aplicadas \\ Universidade Estadual de Campinas - UNICAMP, Limeira-SP, Brasil
}

Recebido 30/11/2020, aceito 21/10/2021

RESUMO

\begin{abstract}
Neste estudo utiliza-se a simulação para avaliar o impacto de diversos cenários de funcionamento do comércio na vigência da pandemia de COVID-19. Foi desenvolvido um modelo de simulação, baseado em Teoria de Filas, que permite relacionar o tempo que os estabelecimentos permanecem abertos, cenários de demanda, medidas de distanciamento social, faturamento, e qualidade de atendimento. Foram propostos cenários para diferentes períodos de abertura do comércio por 4, 6,8 e 10 horas diárias. Os dados de atendimento foram coletados com trabalhadores de estabelecimentos comerciais. Após a execução do modelo de simulação foi possível perceber que um período de abertura maior ou igual a 6 horas diárias atende à necessidade de que o cliente não fique no sistema por mais de vinte minutos.
\end{abstract}

Palavras-chave: Teoria das filas, Comércio, Atendimento ao público.

\begin{abstract}
In this study, simulation is used to assess the impact of different scenarios of trade operation during the COVID-19 pandemic. A simulation model was developed, based on Queuing Theory, which allows relating the time that establishments remain open, demand scenarios, measures of social distance, billing, and quality of service. Scenarios have been proposed for different periods of trade opening for $4,6,8$ and 10 hours a day. The attendance data were collected with workers from commercial establishments. After running the simulation model, it was possible to notice that an opening period greater than or equal to 6 hours a day meets the need for the client to stay in the system for more than twenty minutes.
\end{abstract}

Keywords: Queue theory, commerce, Customer service.

\footnotetext{
* Autor para correspondência. E-mail: rodrigo.gigante@facens.br DOI: 10.4322/PODes.2021.014
}

\footnotetext{
${ }^{1}$ Todos os autores assumem a responsabilidade pelo conteúdo do artigo.
} 


\section{Introdução}

O surgimento do coronavírus (COVID-19) trouxe mudanças para a vida das pessoas devido à adoção de medidas tais como o distanciamento social. Como consequência, todo o comércio das cidades foi fechado por tempo indeterminado como forma de se minimizar o contágio.

Um levantamento realizado pelo Serviço Brasileiro de Apoio às Micro e Pequenas Empresas (SEBRAE) aponta que aproximadamente $4 \%$ das pequenas empresas encerraram suas atividades de maneira definitiva durante a pandemia de COVID-19, 29\% das pequenas e micro empresas continuam com as portas fechadas e $84 \%$ das empresas continuam com redução no faturamento (SEBRAE, 2020).

Muitos dos estabelecimentos de pequeno e médio porte podem simplesmente fechar as portas definitivamente devido ao longo período com baixo ou nenhum faturamento. Durante o período de isolamento social houve queda no faturamento do comércio da ordem de 29,7\% (Cielo, 2020).

A pressão econômica para a retomada do comércio envolve a elaboração de políticas públicas para a reabertura do mesmo. Uma das preocupações é reduzir o tempo de exposição de um consumidor dentro de um estabelecimento comercial, evitando-se a disseminação do vírus (CDC, 2020) (ElPaís, 2020).

Este trabalho tem como objetivo estudar e analisar cenários para a retomada do comércio pós-pandemia de COVID-19, por meio de um modelo de simulação baseado em teoria de filas para análise de cenários. Para o desenvolvimento do estudo foram levantados dados de atendimento através de entrevistas com funcionários e proprietários de estabelecimentos comerciais para a determinação do processo de atendimento.

Entende-se que um fator-chave para garantir a demanda do comércio é aumentar a segurança sanitária do cliente, ou seja, evitar que o mesmo fique muito tempo em um ambiente sujeito à contaminação pelo vírus, conforme estudo do CDC (2020). A forma encontrada para medir essa segurança foi empregar o número de pessoas na fila como fator limitante para o funcionamento do comércio, ou seja, é recomendado que o cliente não permaneça mais que 20 minutos em um ambiente.

\section{Método da Pesquisa}

Um sistema de atendimento irá apresentar filas se, em algum período do dia, a capacidade de atendimento for menor que a demanda. No comércio, em bancos e serviços de atendimento médico de urgência, por exemplo, não é possível distribuir o atendimento dos clientes uniformemente durante o dia para organizar o atendimento.

Um dos sintomas do inadequado dimensionamento de recursos em um sistema de filas é o congestionamento de clientes. Quando o número de clientes esperando por atendimento é elevado, ou seja, há uma grande quantidade de pessoas na fila, é sinal de que o número de atendentes disponíveis no sistema foi dimensionado abaixo do ideal para atendimento.

Um sistema de filas é composto por três partes, sendo elas: (i) uma fonte de usuários, (ii) um ou mais servidores (processadores) em série ou em paralelo e (iii) uma fila na qual os usuários aguardam o atendimento. Em sistemas como um corpo de bombeiros, as filas ficam dispersas geograficamente pelas regiões onde deverá ocorrer o atendimento (Hillier e Lieberman, 2013).

O estudo dos sistemas de filas busca balancear a quantidade de recursos disponíveis para atendimento dos clientes e a demanda, geralmente dinâmica, por atendimento durante a jornada de trabalho. Podem existir limitações de tamanho e espaçamento das pessoas na fila, o número máximo de atendentes que podem estar fisicamente no ambiente, e ainda, a distância que o servidor deve percorrer para chegar ao cliente.

Simulação é um conjunto de instruções com o objetivo de estudar o comportamento de um sistema ao longo do tempo em vários cenários. A simulação desenvolvida fornece métricas do sistema para vários cenários de demanda e assim é possível inferir quais decisões fornecem os 
melhores desempenhos para o sistema (Morgan et al., 1984).

Para a representação do atendimento no comércio foi desenvolvido um modelo de simulação de eventos discretos. Tal modelo considera as lojas do comércio como um sistema de fila única, com capacidade de atendimento de cinco clientes no interior da loja e limitação máxima de clientes na fila, também em cinco clientes, respeitando o distanciamento social. A Figura 1 representa $o$ sistema de filas proposto.

Figura 1: Fluxograma do processo de atendimento.

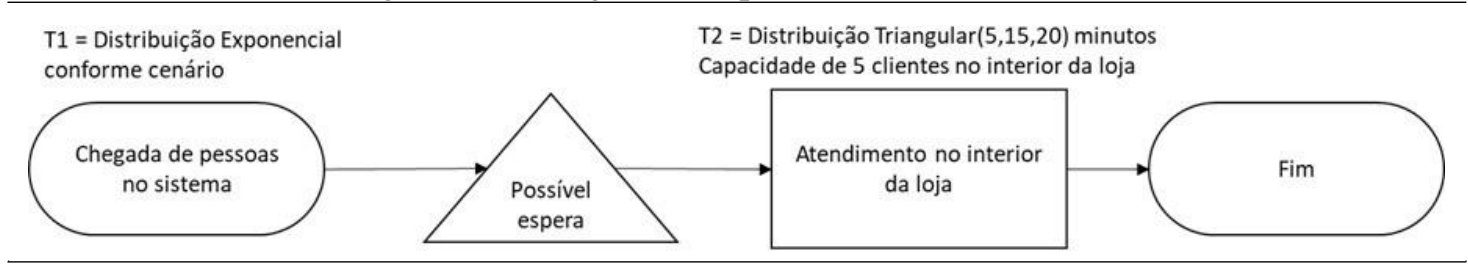

Fonte: Desenvolvido pelos autores.

O tempo (T1) é utilizado para o processo de chegada e varia conforme cenário proposto de abertura do comércio. O tempo (T2) foi coletado a partir de entrevistas com trabalhadores e proprietários de estabelecimentos comerciais nas cidades de Sorocaba e São Carlos.

Para análise de possibilidades de abertura gradual do comércio e os impactos para os clientes, foram analisados os períodos de abertura de 4 horas diárias, 6 horas diárias, 8 horas diárias e 10 horas diárias, e, para analisar as possíveis variações de faturamento, foram coletados dados referentes à venda do comércio em ABRE (2019) e Cielo (2020).

Para efeito de comparação foi utilizada, no experimento, uma distribuição triangular com mínimo 55 reais, moda 95 reais e máximo 200 reais para cada venda no período. Além disso, foram desenvolvidos oito cenários de demanda para cada período de funcionamento com objetivo de criar uma comparação mais ampla das possiblidades de comportamento dos clientes. Foi estabelecido um padrão inicial, cenário 1, de atendimento de 80 clientes por dia. Em seguida, foram devenvolvidas variações deste padrão: o cenário 2 possui um aumento de $20 \%$ de demanda entre os turnos, ou seja, no cenário 2 são esperados 80 clientes para o período de abertura de 4 horas, 96 clientes para o período de abertura de 6 horas, 115 clientes para o período de abertura de 8 horas e 138 clientes para o período de abertura de 10 horas. Segundo o mesmo princípio, parao cenário 3 espera-se o atendimento de 100 clientes durante o dia independentemente do período de abertura e, no cenário 4 um aumento de $20 \%$ em cada período de abertura, 100 clientes para 4 horas de funcionamento, 120 clientes para 6 horas de funcionamento, 144 clientes para 8 horasde funcionamento e 173 clientes para 10 horas de funcionamento, as quantidades de clientes paraos cenários 5 a 8 foram criados seguindo o mesmo princípio. As demandas esperadas de clientes para cada um dos oito cenários criados são apresentadas na Tabela 1.

O modelo de simulação de filas proposto foi implementado no software de simulação a eventos discretos FlexSim versão 2017.0.13, e foi executada a simulação para um dia de trabalho para 20 replicações de cada um dos cenários. Para o cálculo do número de replicações foi utilizada a fórmula proposta por Banks et al. (2014).

$$
n \geq\left(\frac{T_{a / 2} S_{0}}{e}\right)^{2}
$$

sendo que:

- n é o número de replicações necessárias;

- $T_{a / 2}$ representa o valor da tabela t-estudem padrão para $5 \%$ de significância com $n-1$

graus de liberdade;

- $S_{0}$ é o desvio padrão amostral; 
Gigante et al. / 14 (2021), p. 1-12

Tabela 1: Demanda esperada de clientes para cada cenário.

\begin{tabular}{|l|l|l|l|l|}
\hline \multicolumn{5}{|c|}{ Demanda esperada por período } \\
\hline Período de abertura & 4 horas & 6 horas & $\mathbf{8}$ horas & $\mathbf{1 0}$ horas \\
\hline Cenário 1 & 80 & 80 & 80 & 80 \\
Cenário 2 & 80 & 96 & 115 & 138 \\
Cenário 3 & 100 & 100 & 100 & 100 \\
Cenário 4 & 100 & 120 & 144 & 173 \\
Cenário 5 & 150 & 150 & 150 & 150 \\
Cenário 6 & 150 & 180 & 216 & 259 \\
Cenário 7 & 200 & 200 & 200 & 200 \\
Cenário 8 & 200 & 240 & 288 & 346 \\
\hline
\end{tabular}

Fonte: Desenvolvido pelos autores.

- $e$ representa o erro no modelo, assumido como 5\% do valor da média amostral.

Os resultados referentes à aplicação da fórmula para 20 replicações estão compilados e apresentados na Tabela 2.

Tabela 2: Número de replicações necessárias.

\begin{tabular}{|l|l|l|l|l|}
\hline \multicolumn{5}{|c|}{ Número de replicações necessárias } \\
\hline Período de abertura & 4 horas & 6 horas & 8 horas & 10 horas \\
\hline Cenário 1 & 15 & 6 & 3 & 1 \\
Cenário 2 & 13 & 6 & 7 & 3 \\
Cenário 3 & 11 & 6 & 3 & 2 \\
Cenário 4 & 5 & 17 & 9 & 10 \\
Cenário 5 & 4 & 9 & 12 & 2 \\
Cenário 6 & 3 & 5 & 9 & 2 \\
Cenário 7 & 3 & 5 & 9 & 2 \\
Cenário 8 & 3 & 2 & 2 & 1 \\
\hline
\end{tabular}

Fonte: Desenvolvido pelos autores.

Com os resultados da Tabela 2, pode-se perceber que o número de replicações utilizado neste estudo respeita a proposta de Banks et al. (2014). Por exemplo, o tempo médio de espera dos clientes no cenário 4 com 6 horas de funcionamento para 20 replicações foi 17,2 minutos.

Aplicando a fórmula, encontra-se:

$$
n \geq\left(\frac{2,09 \cdot 1,7}{0,86}\right)^{2}=17,06
$$

\section{Resultados}

Para analisar os resultados para cada um dos cenários, foram utilizadas as métricas: número total de clientes atendidos durante o dia; número médio de clientes no sistema; tempo médio de permanência no sistema; taxa de atendimento por hora e faturamento médio diário. A média do total de clientes atendidos por dia em cada cenário e por período de abertura é apresentada na Tabela 3.

Com o aumento do período de funcionamento e o aumento do número de clientes propostos nos cenários, percebe-se um aumento na quantidade de clientes atendidos por dia. O período de funcionamento de 4 horas apresenta uma limitação na quantidade total de clientes atendidos, independente do período de abertura do comércio. Para os períodos de abertura de 6 a 10 horas, o aumento da demanda nos cenários acompanha o aumento do número total de clientes atendidos. 
Gigante et al. / 14 (2021), p. 1-12

Tabela 3: Clientes atendidos no dia.

\begin{tabular}{|l|l|l|l|l|}
\hline \multicolumn{7}{c|}{ Clientes atendidos no dia } \\
\hline Período de abertura & 4 horas & 6 horas & 8 horas & $\mathbf{1 0}$ horas \\
\hline Cenário 1 & 76,7 & 76,1 & 86,2 & 82,8 \\
Cenário 2 & 78,9 & 107,1 & 118,6 & 141,2 \\
Cenário 3 & 86,7 & 97,4 & 101,4 & 101,8 \\
Cenário 4 & 85,8 & 114,9 & 138,9 & 169,4 \\
Cenário 5 & 90,4 & 125,2 & 151,1 & 145,4 \\
Cenário 6 & 91,2 & 130,5 & 173,9 & 213,9 \\
Cenário 7 & 91,6 & 131,8 & 172,9 & 193,0 \\
Cenário 8 & 91,7 & 131,7 & 181,3 & 218,5 \\
\hline
\end{tabular}

Fonte: Desenvolvido pelos autores.

Esta variação pode ser melhor analisada na Figura 2.

Figura 2: Total médio de clientes atendidos por dia em cada cenário.

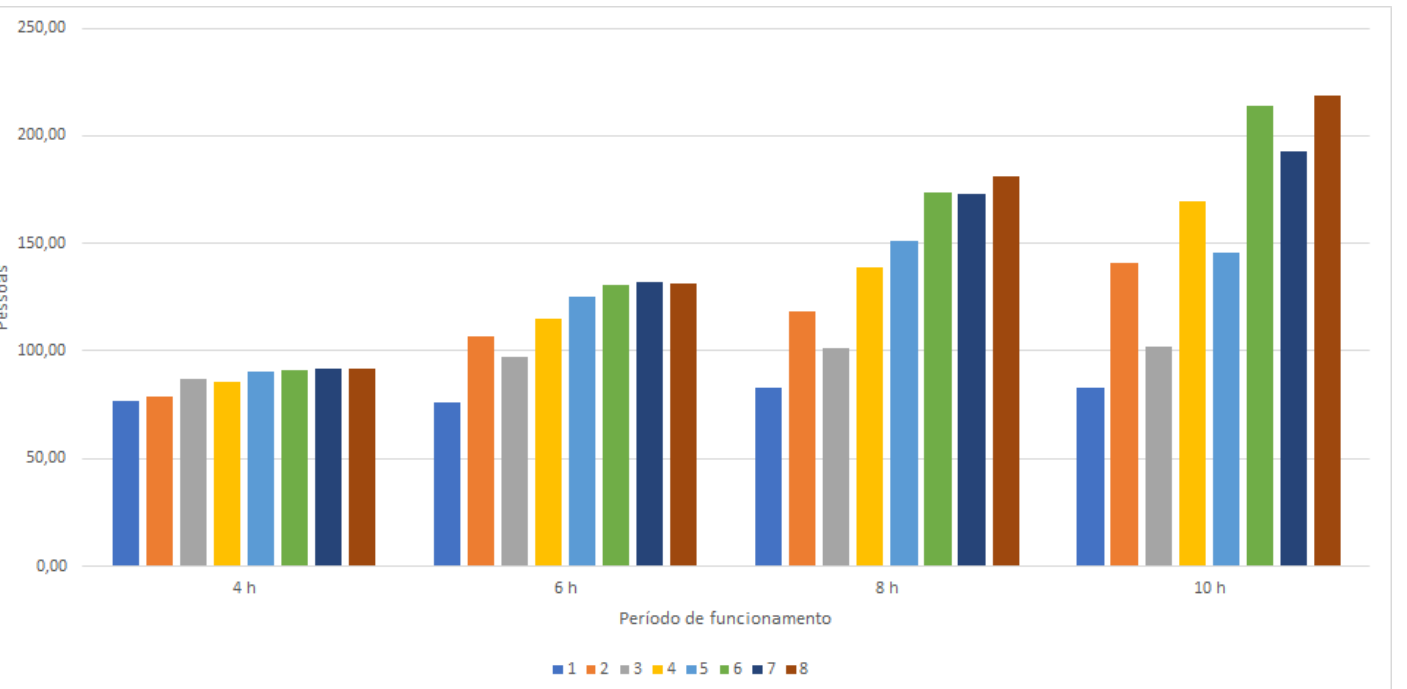

Fonte: Desenvolvido pelos autores.

Na Figura 2 percebe-se que um aumento das quantidades de clientes atendidos para os cenários com o aumento da demanda, apenas os cenários 1 e 3, apresentam atendimento constante, independente do período de abertura, fato condizente com o modelo, pois as demandas esperadas para estes são, respectivamente, 80 e 100 clientes.

Durante o período de pandemia o distanciamento social é fator relevante e, desta forma, a variável que deve ser considerada chave para este estudo é o tempo médio de permanência no sistema. Como fator relevante para o estudo, foi considerado que o tempo médio de permanência no sistema não deve ser superior a 20 minutos, como forma de prevenção ao COVID-19. Os dados referentes ao tempo médio de permanência são apresentados na Tabela 4.

A Figura 3 apresenta o aumento gradual do tempo médio que os clientes permanecem no sistema, tempo em fila mais tempo de atendimento, para realização das compras. É possível perceber o aumento do tempo de permanência no sistema com o aumento da demanda. Este tempo é limitado ao máximo de 23,9 minutos para o cenário 8 por 4 horas de funcionamento. Um ponto a ser destacado é a limitação do número de clientes na fila a cinco pessoas. Com esta medida, em qualquer cenário e em todos os tempos de funcionamento pode-se limitar o tempo que o cliente permanece no sistema fazendo assim com que o isolamento social seja respeitado. Os cenários com melhor resultado no tempo de permanência no sistema são os cenários 1 a 5 , independentemente do tempo de funcionamento. Com isso, se o gerente ou proprietário espera 
uma demanda menor ou igual a 150 clientes, com a limitação de cinco clientes na fila, os clientes não ficarão em fila mais que 20 minutos.

Tabela 4: Tempo médio do cliente no comércio para realização das compras.

\begin{tabular}{|l|l|l|l|l|}
\hline \multicolumn{5}{|c|}{ Tempo médio de permanência no sistema (minutos) } \\
\hline Período de abertura & 4 horas & 6 horas & $\mathbf{8}$ horas & $\mathbf{1 0}$ horas \\
\hline Cenário 1 & 16,7 & 14,0 & 13,6 & 13,6 \\
Cenário 2 & 16,8 & 15,1 & 14,5 & 14,5 \\
Cenário 3 & 19,1 & 15,5 & 13,9 & 13,6 \\
Cenário 4 & 19,5 & 17,2 & 15,4 & 15,7 \\
Cenário 5 & 22,9 & 19,8 & 16,7 & 14,6 \\
Cenário 6 & 22,9 & 21,7 & 20,3 & 20,1 \\
Cenário 7 & 23,7 & 22,3 & 19,3 & 17,3 \\
Cenário 8 & 23,9 & 23,4 & 23,3 & 23,3 \\
\hline
\end{tabular}

Fonte: Desenvolvido pelos autores.

Figura 3: Tempo médio de permanência no sistema.

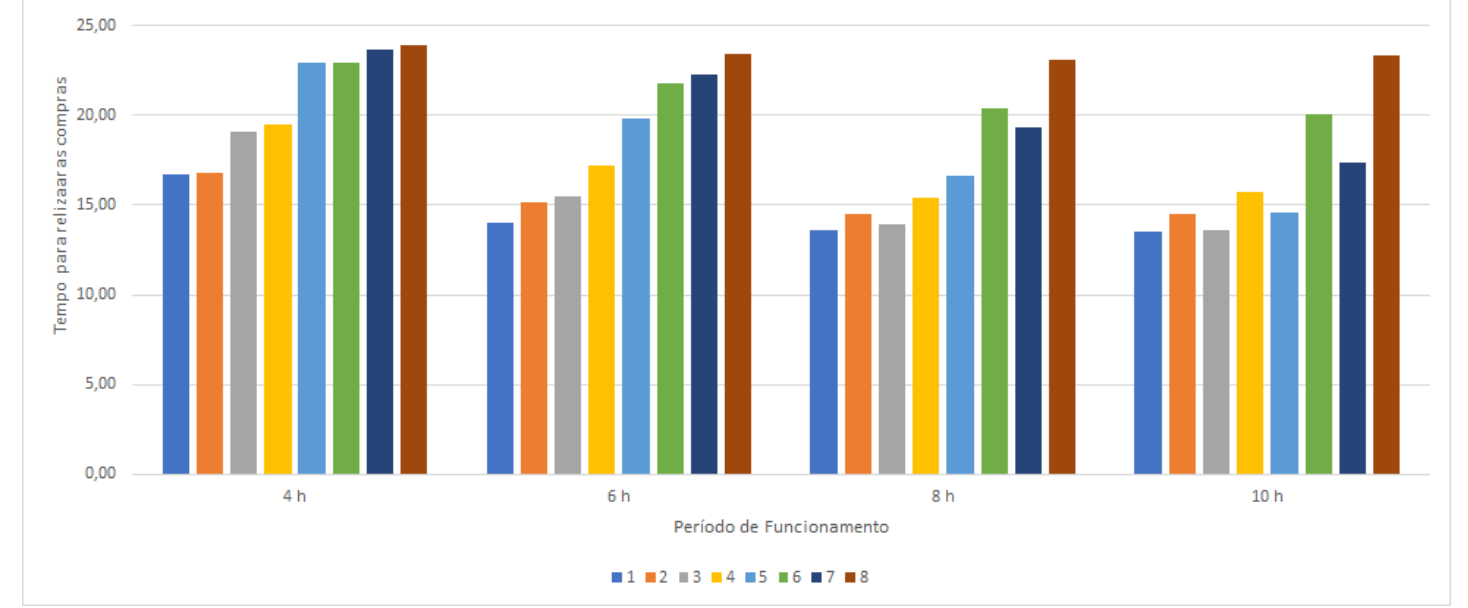

Fonte: Desenvolvido pelos autores.

A métrica de número médio de clientes no sistema é importante para a análise de sistemas de filas, pois indica o congestionamento do sistema, para a pandemia de COVID-19. Esta medida é útil para analisar a quantidade média de pessoas que estarão no sistema durante o período de trabalho. Os resultados do número médio de clientes no sistema são apresentados na Tabela 5.

Tabela 5: Número médio de clientes no sistema.

\begin{tabular}{|l|l|l|l|l|}
\hline \multicolumn{5}{|c|}{ Número médio de clientes no sistema } \\
\hline Período de abertura & $\mathbf{4}$ horas & 6 horas & 8 horas & $\mathbf{1 0}$ horas \\
\hline Cenário 1 & 5,3 & 3,0 & 2,3 & 1,9 \\
Cenário 2 & 5,4 & 3,9 & 3,5 & 3,4 \\
Cenário 3 & 6,8 & 4,3 & 2,9 & 2,3 \\
Cenário 4 & 6,9 & 5,7 & 4,4 & 4,5 \\
Cenário 5 & 8,6 & 7,0 & 5,2 & 3,6 \\
Cenário 6 & 8,6 & 8,1 & 7,3 & 7,3 \\
Cenário 7 & 9,1 & 8,4 & 6,9 & 5,7 \\
Cenário 8 & 9,1 & 8,8 & 8,7 & 8,7 \\
\hline
\end{tabular}


Fonte: Desenvolvido pelos autores.

A Figura 4 apresenta graficamente o aumento do tempo de permanência no sistema com o aumento da demanda. O aumento de tempo não é expressivo pois foi imposto um limite no número de pessoas que podem permanecer na fila.

Figura 4: Número médio de clientes no sistema.

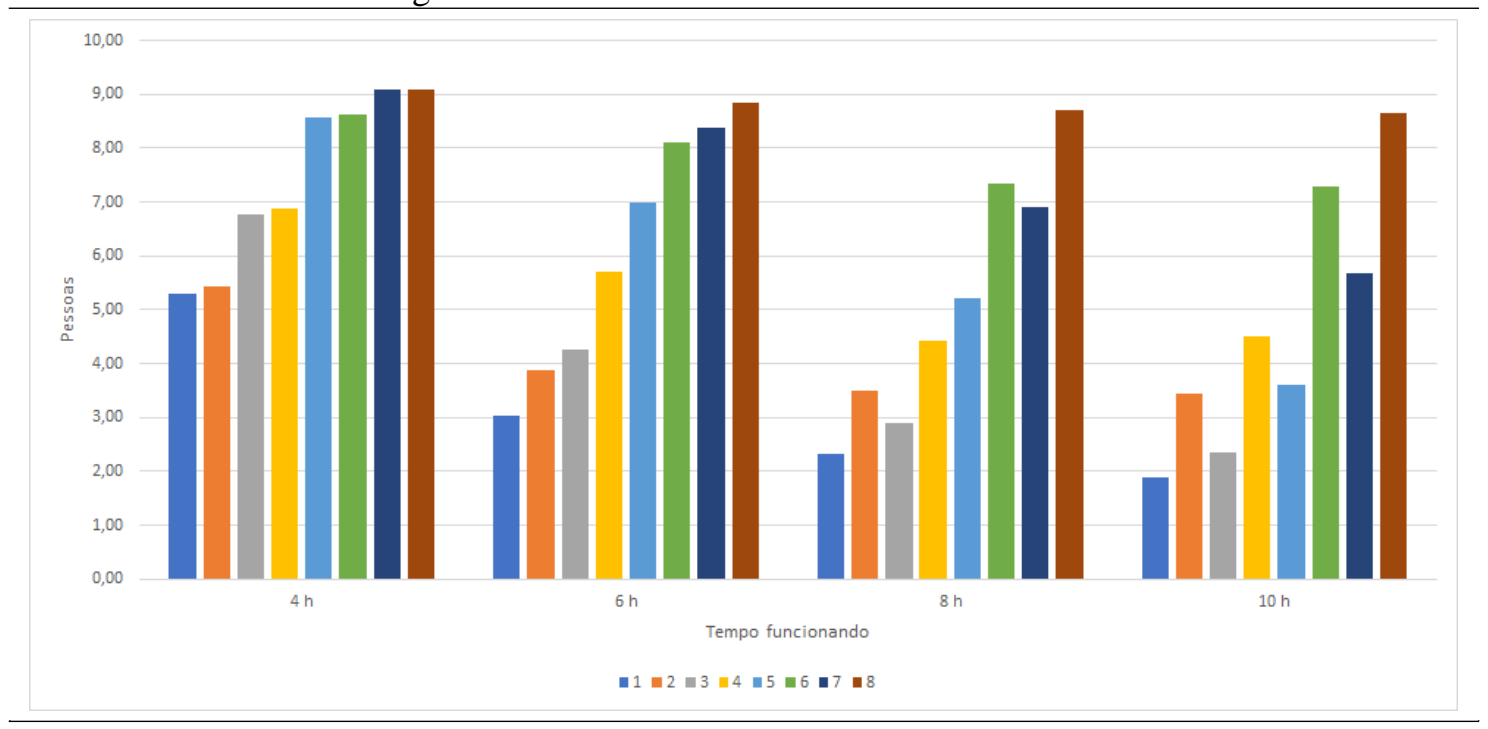

Fonte: Desenvolvido pelos autores.

Com a utilização do fator de segurança de fila máxima de cinco clientes a quantidade de pessoas no sistema é menor para os períodos de abertura maiores que 6 horas e para os cenários 1 a 5. Mais uma vez, caso seja esperada uma demanda menor ou igual a 150 clientes durante o período de funcionamento as restrições de isolamento social são mantidas.

O resultado da limitação do número de clientes na fila é o não atendimento a uma quantidade de clientes por dia. Esta medida foi considerada no estudo e os resultados estão apresentados na Tabela 6 .

Tabela 6: Número médio de clientes não atendidos.

\begin{tabular}{|l|l|l|l|l|}
\hline \multicolumn{5}{|c|}{ Número médio de clientes não atendidos } \\
\hline Período de abertura & 4 horas & 6 horas & $\mathbf{8}$ horas & $\mathbf{1 0}$ horas \\
\hline Cenário 1 & 4,15 & 0,4 & 0,0 & 0,0 \\
Cenário 2 & 3,1 & 0,7 & 0,7 & 0,4 \\
Cenário 3 & 13,5 & 1,1 & 0,1 & 0,0 \\
Cenário 4 & 12,3 & 6,0 & 1,9 & 3,7 \\
Cenário 5 & 56,9 & 21,9 & 5,3 & 0,8 \\
Cenário 6 & 58,9 & 48,8 & 37,9 & 37,7 \\
Cenário 7 & 101,3 & 63,8 & 25,3 & 9,6 \\
Cenário 8 & 106,2 & 98,10 & 105,3 & 121,65 \\
\hline
\end{tabular}

Fonte: Desenvolvido pelos autores.

O aumento do número de clientes que não podem ser atendidos é maior para os cenários com mais de 150 clientes com período de funcionamento menor a 6 horas. Desta forma, caso o responsável espere uma demanda inferior a 150 pessoas e esteja funcionando por 6 ou mais horas, irá deixar de atender, no máximo, 6 clientes, conforme apresentado na Tabela 6. A diferença nas quantidades de clientes não atendidos podem ser melhor visualizadas na Figura 5.

Para analisar os impactos do período de abertura e o número de clientes atendidos em 
relação ao faturamento médio dos estabelecimentos, foi considerado que cada cliente irá realizar uma compra conforme uma distribuição triangular com mínimo 55, moda 95 e máximo 200 reais em todos os cenários. Desta forma, é possível verificar o aumento do faturamento com o aumento do número de clientes atendidos no dia. Os resultados para cada um dos cenários são apresentados na Tabela 7.

Figura 5: Número médio de clientes não atendidos em cada cenário.

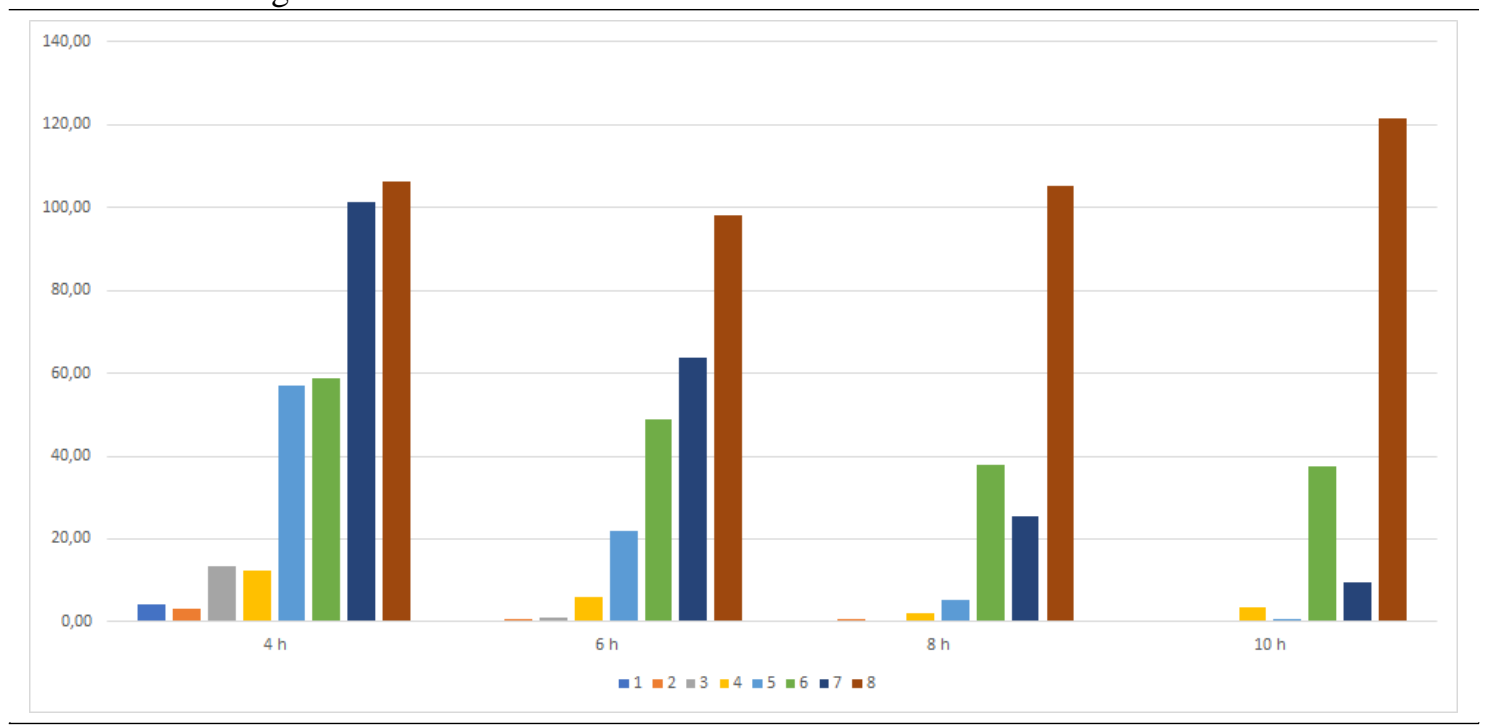

Fonte: Desenvolvido pelos autores.

Tabela 7: Faturamento médio por cenário.

\begin{tabular}{|l|l|l|l|l|}
\hline \multicolumn{5}{|c|}{ Faturamento médio por cenário } \\
\hline Período de abertura & $\mathbf{4}$ horas & 6 horas & 8 horas & $\mathbf{1 0}$ horas \\
\hline Cenário 1 & $8.728,52$ & $8.716,13$ & $9.529,27$ & $9.562,84$ \\
Cenário 2 & $9.094,22$ & $10.617,86$ & $13.618,02$ & $16.182,34$ \\
Cenário 3 & $9.864,94$ & $11.261,00$ & $11.636,99$ & $11.755,38$ \\
Cenário 4 & $9.987,74$ & $13.191,53$ & $15.847,63$ & $19.579,83$ \\
Cenário 5 & $10.341,06$ & $14.425,27$ & $17.368,07$ & $16.732,14$ \\
Cenário 6 & $10.531,17$ & $15.077,75$ & $20.046,19$ & $24.645,16$ \\
Cenário 7 & $10.636,67$ & $15.176,23$ & $19.901,26$ & $22.407,97$ \\
Cenário 8 & $10.589,99$ & $15.187,03$ & $20.780,39$ & $25.138,67$ \\
\hline
\end{tabular}

Fonte: Desenvolvido pelos autores.

Na Tabela 7 nota-se que a limitação na quantidade de clientes atendidos no período de funcionamento de 4 horas faz com que o faturamento seja limitado, independentemente de qual cenário de demanda ocorra. O responsável pelo estabelecimento deve identificar qual o seu perfil de demanda esperado, por exemplo, se são esperados 100 clientes em um dia, independente do tempo de funcionamento, situação descrita no cenário 3, o faturamento irá variar entre $\mathrm{R} \$ 9.864,94$ e R \$ 11.755,38, devem ser considerados, antes da decisão, os custos envolvidos em ampliar o per'iodo de funcionamento de 4 para 10 horas ira' gerar um aumento de faturamento menor que $\mathrm{R} \$$ $1.000,00$. A decisão de um período de funcionamento maior deve ser analisada com cautela, pois, neste momento, muitos dos clientes não voltaram às compras.

Para uma análise da capacidade dos sistemas de filas a taxa de atendimento por hora foi estudada para cada um dos cenários estudados. Os dados referentes ao total de clientes atendidos por hora são apresentados nas Figuras 6 a 13. 
Figura 6: Clientes Atendidos por hora cenário 1.

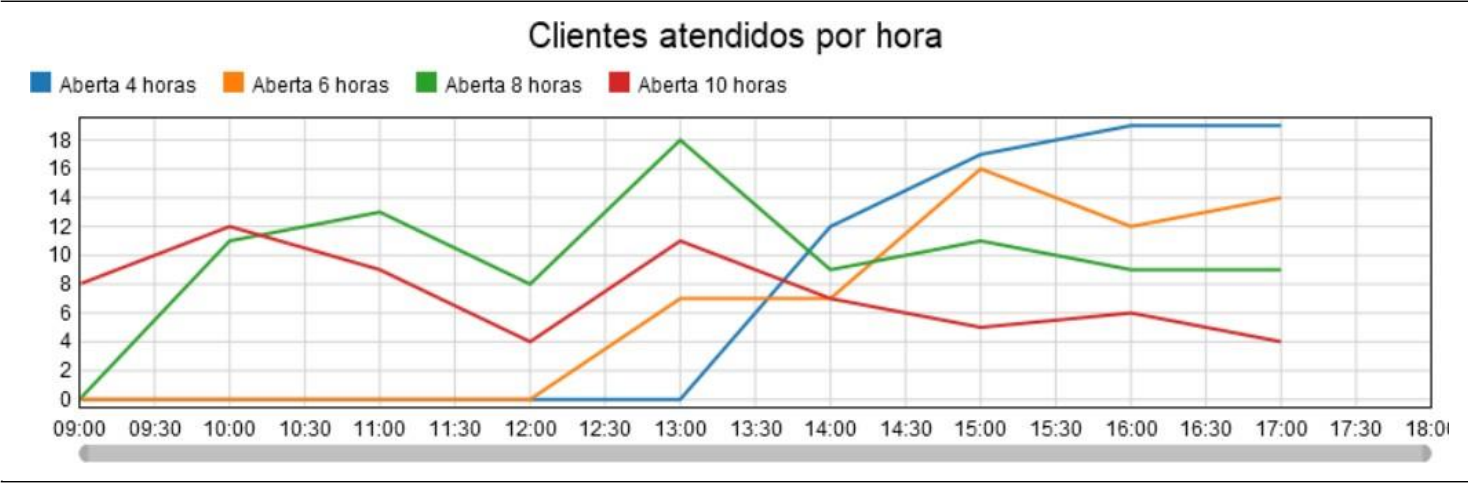

Fonte: Desenvolvido pelos autores.

Figura 7: Clientes Atendidos por hora cenário 2.

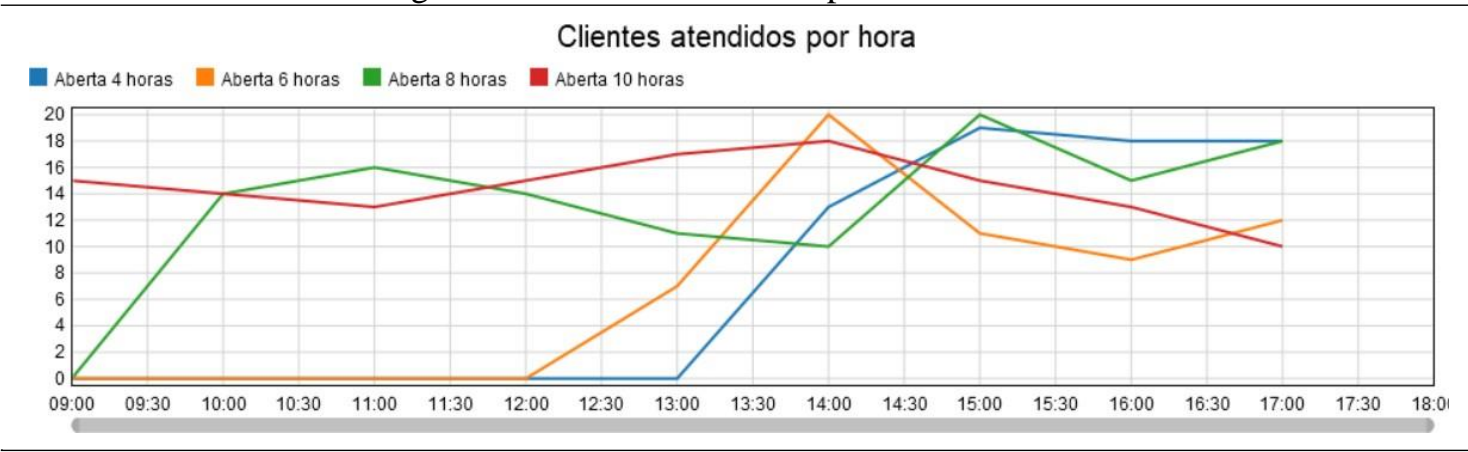

Fonte: Desenvolvido pelos autores.

Figura 8: Clientes Atendidos por hora cenário 3.

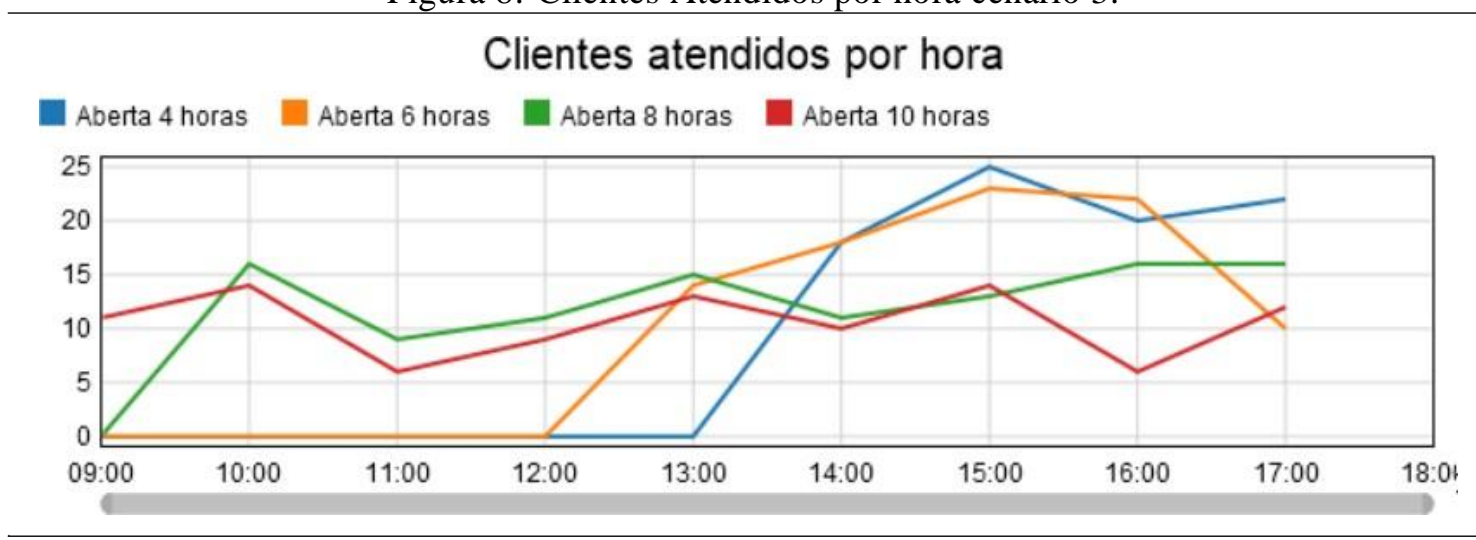

Fonte: Desenvolvido pelos autores.

As figuras 6 a 13 apresentam o número de clientes atendidos por hora no decorrer do dia para cada um dos períodos de atendimento propostos em cada um dos cenários desenvolvidos, respectivamente 1 a 8 . Em todos os cenários não serão atendidos mais que 25 clientes por hora, para qualquer perfil de demanda. Para os cenários com menor demanda prevista, 1 a 4, um período de funcionamento maior cria uma taxa de atendimento menor, que é compensada pela quantidade maior de horas trabalhadas. Dentre estes, considerando os períodos de abertura de 8 e 10 horas, o fluxo de clientes é constante durante o dia, não apresentando acúmulo de clientes para os cenários 1 a 5. 
Gigante et al. / 14 (2021), p. 1-12

Figura 9: Clientes Atendidos por hora cenário 4.

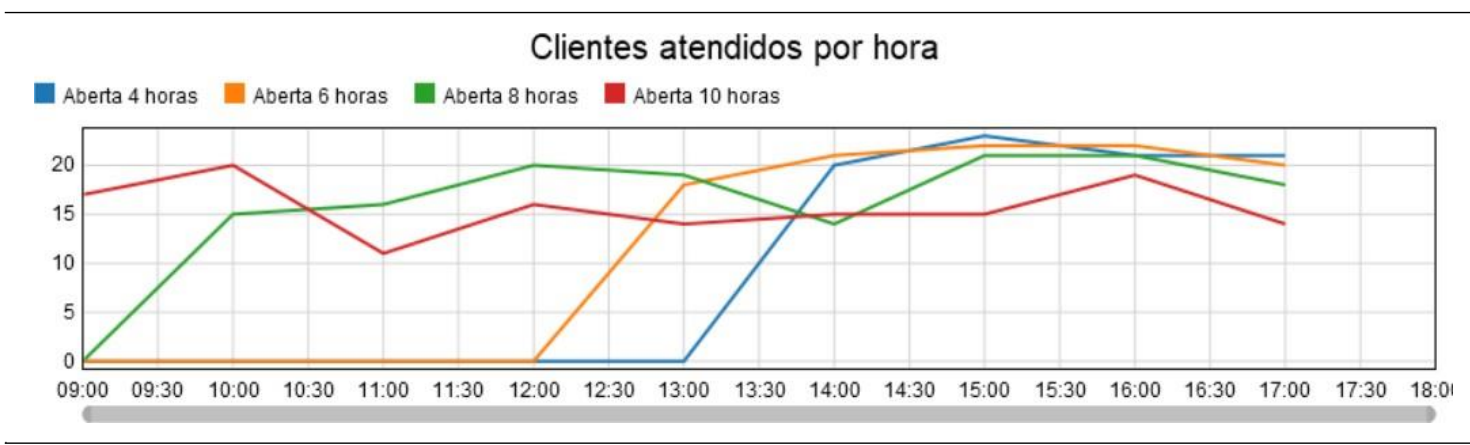

Fonte: Desenvolvido pelos autores.

Figura 10: Clientes Atendidos por hora cenário 5.

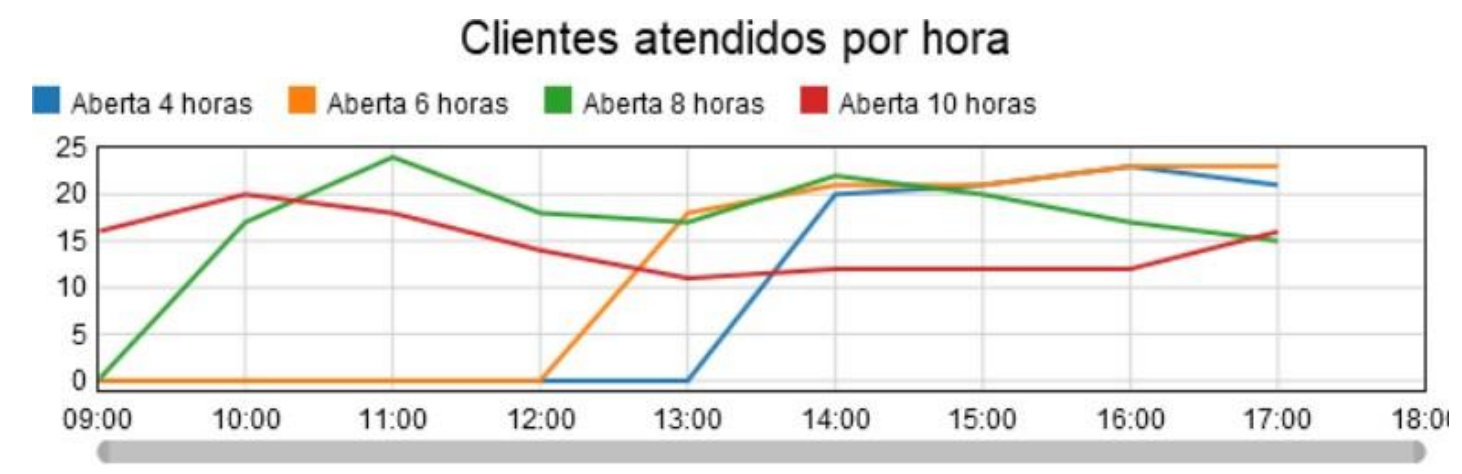

Fonte: Desenvolvido pelos autores.

Figura 11: Clientes Atendidos por hora cenário 6.

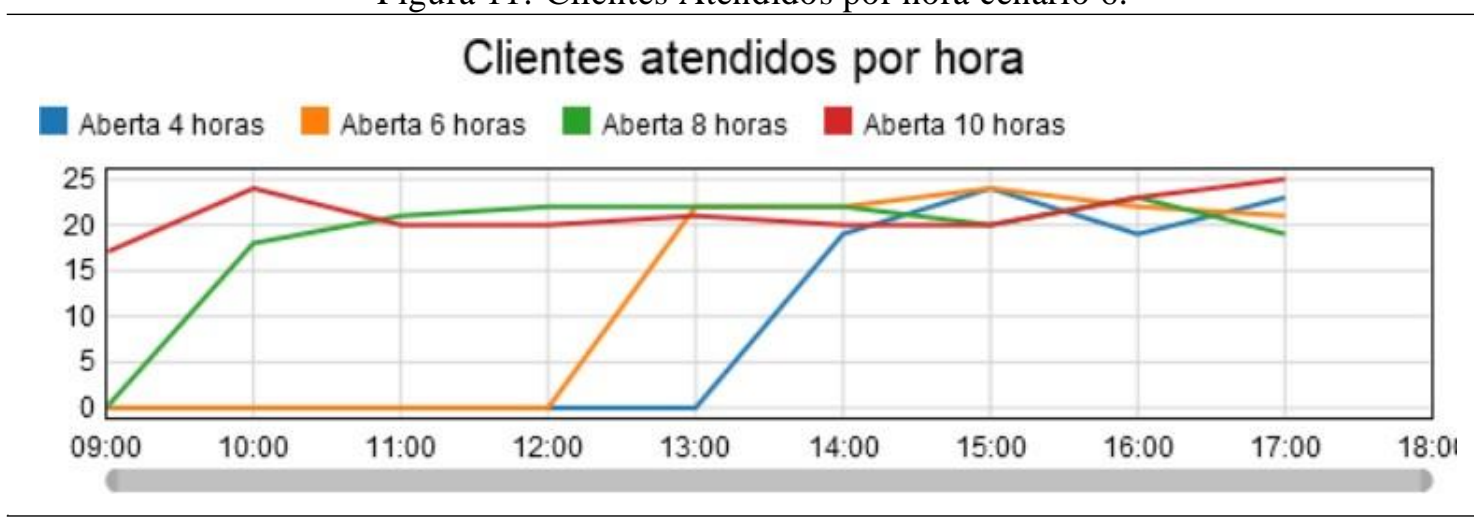

Fonte: Desenvolvido pelos autores.

Analisando as Tabelas 4 e 5, é possível identificar que, para os períodos de abertura a partir de 6 horas, isto é, 6,8 e 10 horas, os clientes apresentam pouco tempo de permanência no sistema. E a quantidade de clientes presentes no sistema também é menor. Da mesma forma analisando a Tabela 7, percebe-se o aumento de faturamento, devido ao aumento do número de clientes atendidos, para os cenários com tempo de funcionamento 6,8 e 10 horas. Entretanto, deve-se lembrar que o aumento do número de horas trabalhadas poderá gerar aumento de custos para o estabelecimento, sendo necessária uma análise detalhada por parte do gestor antes da decisão do número de horas que estará trabalhando. 
Gigante et al. / 14 (2021), p. 1-12

Figura 12: Clientes Atendidos por hora cenário 7.

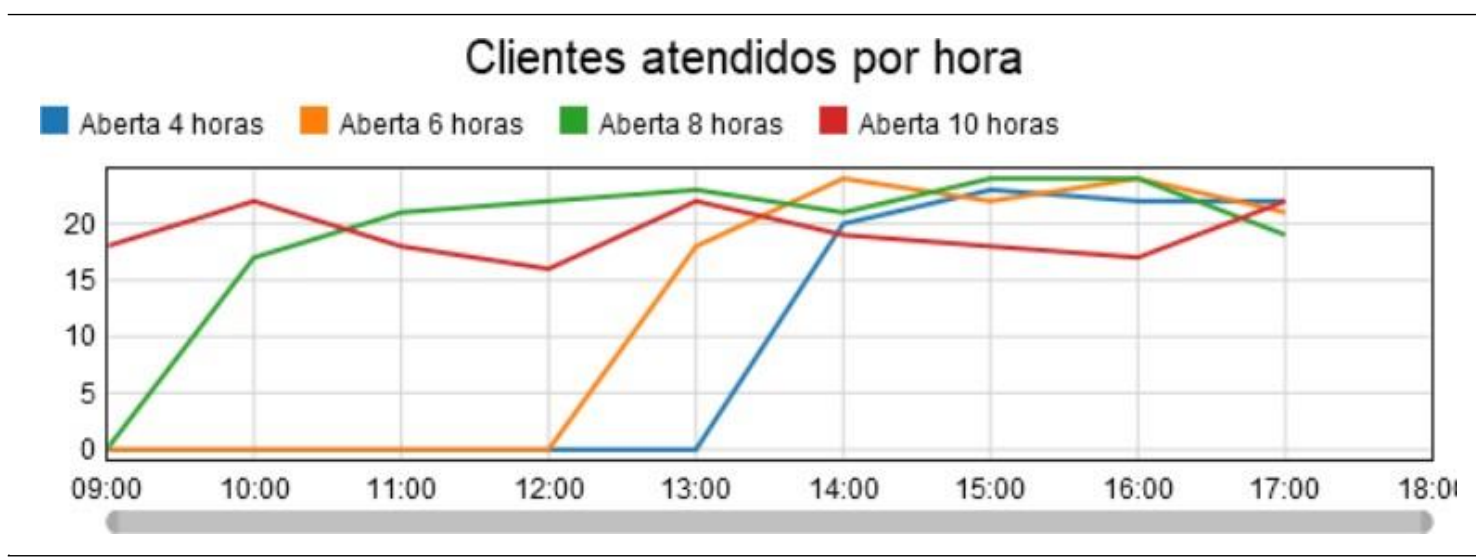

Fonte: Desenvolvido pelos autores.

Figura 13: Clientes Atendidos por hora cenário 8.

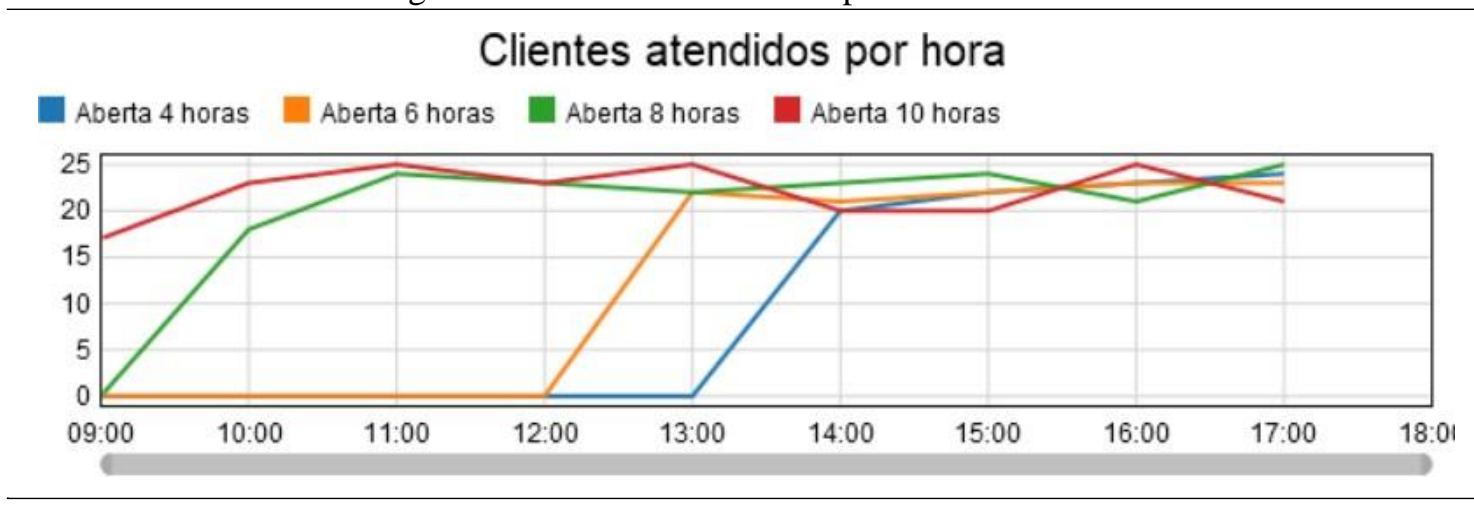

Fonte: Desenvolvido pelos autores.

\section{Conclusão}

Os resultados apresentados demonstram que, para um tempo de permanência no sistema baixo, o tempo de abertura dos estabelecimentos pode ser de 4 horas para os cenários 1 a 4 , ou seja, a demanda esperada é menor ou igual a 150 clientes. Para demandas por clientes maiores, cenários 6 e 7, o tempo de funcionamento deve ser de 8 ou 10 horas para que seja respeitado o tempo de permanência de 20 minutos. No cenário de demanda 8 , sempre irá ocorrer o tempo médio de permanência no sistema maior que 20 minutos.

Os indicadores tempo médio de permanência no sistema, número médio de clientes em espera, número médio de clientes atendidos e taxa de clientes atendidos por hora foram melhores para cenários com demandas inferiores a 173 clientes por dia (cenário 4), independentemente do tempo de funcionamento do estabelecimento. Desta forma, qualquer que seja o período de funcionamento determinado pelas autoridades o isolamento social será respeitado se a demanda por clientes não for elevada, o que remete à aceitação do isolamento por parte da população.

$\mathrm{O}$ faturamento médio apresentado na Tabela 7 tem como objetivo ser um direcionador da variação dos lucros que serão gerados com a variação do tempo de abertura. $O$ gestor deve analisar seu perfil de demanda esperado e utilizar a tabela como base para decisões operacionais.

Este estudo apresentou um direcionamento para os comerciantes e gestores públicos analisarem sugestões de como pode ser dar o processo de abertura gradual dos estabelecimentos comerciais, conforme a demanda das cidades, respeitando o isolamento social e observando uma estimativa de faturamento para os comerciantes. 
Gigante et al. / 14 (2021), p. 1-12

Como proposta de trabalho futuro, sugere-se análise detalhada dos tipos de demanda por característica do estabelecimento, atendimento em salões de beleza e barbearias e a análise do período de abertura e ampliação do faturamento conforme características dos estabelecimentos.

Agradecimentos. Os autores agradecem o apoio da Sociedade Brasileira de Pesquisa Operacional (SOBRAPO).

\section{Referências}

ABRE. Como foram as vendas em dadas comemorativas em 2019. 2019. Disponível em: https://www.abre.org.br/inovacao/varejo/varejo-em-2019-como-foram-as-vendas-nasprincipais-datas-comemorativas-do-ano/. Acessado em: 01/08/2020.

Banks, J., Carson II, J. S., Nelson, B. L. e Nicol, D. M. Discret-Event System Simulation. USA: Pearson, 2014.

CDC. How covid-19 spreads. 2020. Disponível em: https://www.cdc.gov/coronavirus/2019ncov/prevent-getting-sick/how-covid-spreads.html. Acessado em: 16/07/2020.

Cielo. Impacto do covid-19 no varejo brasileiro. 2020. Disponível em: https://www.cielo.com.br/boletim-cielo-varejo/. Acessado: 16/07/2020.

ElPaís. Radiografia de três surtos de coronavírus: como se infectara e como podemos evitar. 2020. Disponível em: https://brasil.elpais.com/brasil/2020-06-16//radiografia-de-tres-surtos-decoronavirus-como-se-infectaram-e-como-podemos-evitar.html. Acessado em: 16/07/2020.

Hillier, F. S. e Lieberman, G. J. Introdução à Pesquisa Operacional. Porto Alegre-RS: Mc Graw Hill/Bookman, 2013.

Morgan, C. B., Banks, J. e Carson, J. S. Discrete-Event System Simulation. USA: Pearson, 1984.

SEBRAE. Impactos da covid-19 nos pequenos negócios. 2020. Disponível em: https://datasebrae.com.br/wp-content/uploads/2020/07/Resumo-Setorial_n5-v2_compressed1.pdf. Acessado em: 16/07/2020. 